\title{
Risk Factors for Superficial Side Infections after Total Hip Arthroplasty
}

\author{
Eder Annabelle ${ }^{1}$, Windhager Reinhard ${ }^{1}$, Pilz Veronika ${ }^{2}$, Hanstein Tim ${ }^{2}$, Kühn Klaus-Dieter ${ }^{3, *}$ \\ ${ }^{1}$ Department of Orthopaedic Surgery, Medical University of Vienna, the General Hospital, Austria \\ ${ }^{2}$ Heraeus Medical GmbH, Wehrheim, Germany \\ ${ }^{3}$ Department of Orthopedics and Trauma Surgery, Medical University of Graz, Austria
}

Copyright $\mathrm{C} 2018$ by authors, all rights reserved. Authors agree that this article remains permanently open access under the terms of the Creative Commons Attribution License 4.0 International License

\begin{abstract}
As the numbers of primary total joint replacements and following revision operations are constantly rising, there are more and more patients with severe comorbidities, which might be considered as additional risk factor for developing a periprosthetic joint infection (PJI). In this study, we analyzed patient-related risk factors, which might influence the occurrence of superficial surgical side infections (SSI) after total hip replacements. There is no study yet, focusing only on superficial SSI rather than on deep infections or PJI in general. Methods: We performed a literature research in Pubmed. Used key words were "hip AND (arthroplasty OR replacement) AND infection." We found 4,417 studies related to this broad definition. After exclusion by defined criteria, 10 relevant studies were left. Results: We found the following patient-related risk factors for superficial SSI: Obesity, age, ASA class $>2$, diabetes mellitus, HIV, hypertension, chronic obstructive pulmonary disease (COPD), previous cardiac surgery, dyspnea, corticosteroid use and hepatitis C. Gender, race and chronic kidney disease were not confirmed as risk factors for superficial SSI. Conclusions: To guarantee the best possible outcome after the surgery, it is advisable to treat these patients before performing a total hip replacement - if possible.
\end{abstract}

Keywords Superficial Surgical Side Infections (SSI), Total Hip Replacement (THA), Comorbidities, Patient Related Risk Factors

\section{Introduction}

Periprosthetic joint infections (PJI) are one of the most devastating complications after total hip replacements [1]. Infections occur in up to $1 \%$ after primary total hip replacement [2] and can be defined as superficial, applying to the skin or subcutaneous tissues [3], and deep infections, applying to the fascia and the muscle tissue [4]. In both cases, the affected patient has to undergo further procedures, ranging from superficial wound cleansing or debridement and implant retention up to several following operations with extensive soft-tissue and bone debridement with removal of the implant and re-implantation after curing the infection. In any case, antibiotic therapy is required to support the surgical approach [5].

Along with the expected increase of total hip replacements in an ageing society [6], the number of deep and superficial surgical site infections (SSI) can be expected to rise as well. Since infection is a severe complication and a major cause of failure after total joint arthroplasty, strategies must be developed to minimize the risk. The major countermeasure against deep and superficial infection is prevention, inter alia by elimination of known risk factors that can be patient-related, surgery-related, inpatient postoperative, and long-term factors [7].

Patient-related risk factors leading to infection have previously been investigated by Eka and Chen [8], and later by Jesenko et al.[9]. However, the former focused their research on risk factors involved in infection of both the hip and the knee and did not distinguish between deep and superficial infection, while the later investigated deep PJI after THA.

Here, we attempted to find patient-related risk factors influencing exclusively the rate of superficial SSI in patients after THA. To identify and analyze these factors, we reviewed the literature published between 1950 and 2014.

\section{Materials and Methods}

The data was received based on a literature search in Pubmed. To avoid falsification, a second database, Embase, was used to compare the results. We used the same key words as Jesenko et al. [9]: "hip AND infection AND (arthroplasty OR replacement)". The relevant studies for 
our research were published between 1950 and 2014. Data published after the year of 2014 were not included.

For reasons of coherence, we applied the same methodology as used by Jesenko et al. [9]. Thus, we followed the same criteria for the screening of the studies but adapted them to superficial infection instead of deep PJI:

1. Language: The studies had to be published in English or German.

2. Inappropriate study type: Animal studies, in vitro studies, no comments or editorials. The study design of the relevant data had to be an original study including case control or cohort groups which could be compared with each other.

3. Studies of other joints than the hip were excluded.

4. The outcome of the study had to be superficial SSI of the hip, other complications were excluded.

5. Studies that did not report person-related risk factors were excluded. Patient related risk factors that could not directly be influenced by the clinic staff were the primary outcome measures.

6. If the full text of a study was not available, the study was excluded.
7. Studies that reported risk factors in combination with another site (e.g. knee) were excluded, since the combination could bias the results.

8. Studies that investigated other procedures in combination with primary THA were excluded.

9. The patient population had to consist of patients who received a primary THA. Patients with a hemiarthroplasty or an already revised hip-replacement are believed to have a different initial situation as regards the outcome of the operation and the risk of developing PJI.10. Although Jesenko et al.[9] did not define the level of significance as a criterion, we decided to include only studies with $\mathrm{p}<$ 0.05 .

\section{Results}

In total, we found 4,417 studies that were published between 1950 and 2014. After applying the inclusion and exclusion criteria, 10 relevant studies were left for review (Table 1). Eleven conditions were reported as risk factors for superficial SSI in patients after THA, and three conditions investigated as potential risk factors were not confirmed.

Table 1. Patient-related risk factors (plus sign) and no risk factors (minus sign) sorted by number of patients

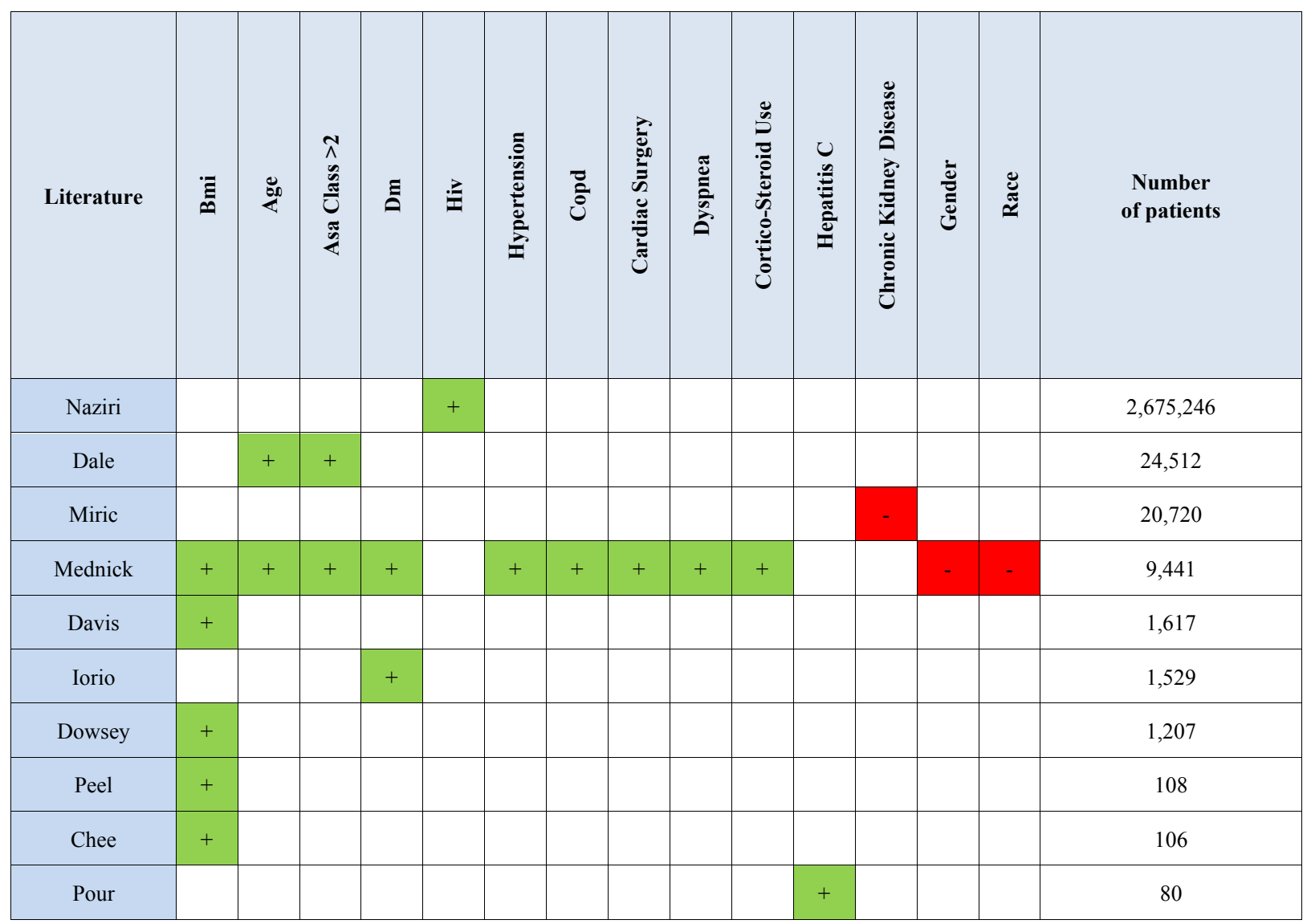


Figure 1 displays each risk factor in a hierarchical order. With five studies in total, we found considerable evidence for BMI as risk factor for superficial joint infections. The clearest result was found in Peel et al. [17] with a significant odds ratio (OR) of 5.0 (95\% CI $1.6-15.9)$. In addition, Davis et al. (95\% CI 1.494 to 7.583 ) [14], Mednick et al. (10.72\% case group vs. $6.39 \%$ control group, $\mathrm{p}<0.001)$ [13], Dowsey et al. $(\mathrm{p}<0.002)[16]$, and Chee et al. $(p=0.012)[18]$ found a significantly higher rate of superficial infections in patients with $\mathrm{BMI} \geq 35 \mathrm{~kg} / \mathrm{m}^{2}-\geq 40$ $\mathrm{kg} / \mathrm{m}^{2}$ compared to patients with lower BMIs.

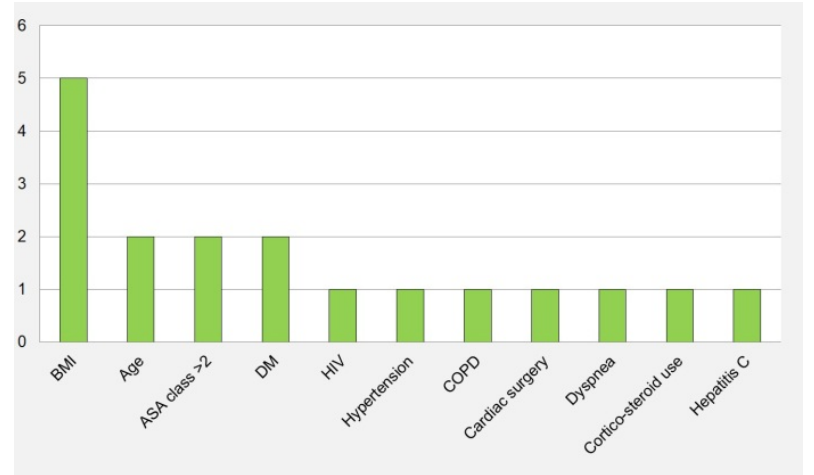

Figure 1. Number of papers for each risk factor. The risk factors are shown in hierarchical order. The numbers display the number of studies, in which the risk factors were shown as a significant risk factor for superficial infection.

Two studies, Mednick et al. (64 vs. 66 years, p < 0.001)[13] and Dale et al. ( $\mathrm{p}=0.03 ; 36 / 816$ CI $1.1-3.5$; 4.2 adjusted 1 year SSI\%)[11], found a relation between the age of the patients and the incidence of superficial SSI in total hip replacements. Both significant younger[13] and older[11] patients than the average age in the cohorts had a significantly higher risk of developing a superficial infection after a total hip replacement. Both studies also identified an American Society of Anesthesiologists (ASA) score above two $(p<0.001[13]$ and $p=0.04[11])$ as risk factors for superficial SSI.

Further, Mednick et al. ( $<<0.001 ; 18.26 \%$ vs. $10.67 \%)$ [13] described diabetes mellitus as risk factor for superficial SSI. This is in line with Iorio et al. $(p<0.00013$; $2.4 \%$ vs. $0.2 \%$ )[15], who found a higher appearance of postoperative superficial infections in patients with Diabetes Mellitus as well.

Other risk factors were found to be human immunodeficiency virus (HIV) (OR: 2.38; 95\% CI: $1.32-$ $4.30 ; \mathrm{p}=0.004)[10]$, hypertension $(66.38 \%$ vs. $56.46 \%$; $<0.001)[13]$, chronic obstructive pulmonary disease (COPD) $(8.70 \%$ vs. $4.12 \%$; p $<0.001)[13]$, previous cardiac surgery $(3.19 \%$ vs. $1.23 \% ; \mathrm{p}=0.002)[13]$, dyspnea $(11.01 \%$ vs. $6.19 \% ; \mathrm{p}=0.001)[13]$, corticosteroid use $(8.41 \%$ versus $3.38 \% ; \mathrm{p}<0.001)[13]$ and hepatitis $\mathrm{C}(\mathrm{p}=$ $0.03)$ [19].

Miric et al.[12] investigated chronic kidney disease as potential risk factor for superficial SSI after THA. They found no predisposition for superficial infection related to this medical condition (HR: 1.13; CI: $0.49-2.60 ; \mathrm{p}=$ $0.771)$.

In addition, race $(p=0.880)$ was discarded as potential risk factor by Mednick et al.[13]. Further, the later found no evidence that gender $(\mathrm{p}=$ 0.983 ) has an impact on superficial SSI frequency.

\section{Discussion}

This study was aiming to establish a current list of patient-related risk factors that predispose THA patients to superficial infections, thereby providing an opportunity for patient and surgeon to develop individual countermeasures and reducing the likelihood of superficial infection. Following our research criteria, we found 10 studies investigating fourteen risk factors potentially related to superficial infections.

Five studies describe that obese patients with a BMI of BMI $\geq 35 \mathrm{~kg} / \mathrm{m}^{2}$ to $\mathrm{BMI} \geq 40 \mathrm{~kg} / \mathrm{m}^{2}$ had a significantly higher rate of superficial infections after THA. This is in line with previous studies, which reported an increased risk of (deep) PJI for obese patients after THA and TKA as well[7-9]. Various issues can be involved for the predisposition in obese patients. The risk of infection increases due to associated comorbid conditions (e.g. ischemic heart disease, hypertension, hypercholesterolemia, poor nutritional status, type two diabetes mellitus), prolonged operative time, increased need for allogeneic blood transfusion, poor oxygenation of the subcutaneous fat tissue, insufficient prophylactic antibiotic dosing and/or postoperative surgical wound complications.

We found two studies that reported a relation between the age of the patients and the incident of superficial infections in THA. Interestingly, Jesenko et al.[9] found only one publication[20] that suggested an age dependent elevated relative risk for developing a PJI after THA, and thus concluded that there are no correlations between age and infection rates. While age was not mentioned at all by Eka and Chen [8] as patient-related risk factor for PJI of the hip and knee, Zmistowski and Alijanipour[7] identified a correlation between age and an increased rate of PJI after total joint arthroplasty. Moreover and similar to our results, the latter found that there seems to be a bimodal distribution, with the lowest PJI incidence in 55-74 year old patients and significantly higher probability of infection in patients older than 75 (OR: 1.28) or younger than 55 (OR: 1.34). The reasons for this bimodal distribution have not yet been defined, but it seems likely that there are different reasons for the elevated rates in younger and older cohorts.

An ASA class above two was found to be a risk factor for superficial SSI by two studies. Similar results were found by Jesenko et al.[9], who describes three studies that evaluated an ASA class of 3 and above as risk factors for deep PJI. Although a relationship between incidence of PJI and higher ASA scores was described by Zmistowski and Alijanipour [7] as well, they emphasized that the ASA 
score should be seen critically regarding its reliability and validity.

Further, we found two studies identifying diabetes mellitus as a positive risk factor for superficial infection. Again, these findings are in concordance with the data for (deep) PJI of the hip and knee [7-9]. Manifold reasons can be responsible for the predisposition in patients with diabetes mellitus, such as impaired wound healing due to macrovascular implications, inferior innate immune responses, reduced function of neutrophil granulocytes and reduced intracellular bactericidal activity. However, several studies have indicated that the risk of infection substantially decreases when glucose blood levels are under control. That is, if HgbAlc values about $7-8 \%$ are met preoperatively.

Mednick et al. [13] investigated 9.441 patients and found no differences in superficial SSI predisposition related to the gender. However, Zmistowski and Alijanipour [7] found some reports indicating an increased incidence for male gender to PJI susceptibility after total joint arthroplasty, while others describe higher rates of PJI in females or could not confirm a link between gender and PJI at all. Jesenko et al. [9] found six out of seven studies reporting a higher infection risk for men after THA. Unfortunately, in these studies the differences between the genders were examined only secondarily.

Males and females differ regarding their sex hormones and sexual chromosome genetic content, which both affect the immune system. Gender-dependent differences that could potentially impact infection prevalence are skin thickness, $\mathrm{pH}$, sebum induction, fat distribution and metabolism rate. However, due to the conflictive findings, it remains unclear if gender affects infection rate after total joint arthroplasty.

Regarding HIV, Naziri et al.[10] showed that patients with HIV have a predisposition towards superficial infection after THA. HIV therapy has improved over the recent years, resulting in a prolonged life-expectancy. Owing to this development, more HIV patients present for total joint arthroplasty. Zmistowski and Alijanpour reported that there is no evidence demonstrating that HIV-positivity per se (meaning in the absence of other confounding risk factors such as intravenous drug use or hemophilia with frequent self-injections) increases the risk of late hematogenous PJI[7]. However, if HIV-positivity is accompanied by hemophilia, the risk of infection increases. Thus, discrepancies in the literature may be related to confounding influence of hemophilia. Jesenko et al.[9] did not report about HIV as a possible risk factor for deep PJI after $\mathrm{TH}$.

Similar to HIV, it was suggested that hepatitis C infection might only be an issue in hemophilic patients [7]. Nevertheless, we found one study reporting about Hepatitis $\mathrm{C}$ as a potential risk factor in patients without hemophilia [19]. The cohort analyzed contained only 40 hepatitis C patients, though.

We further found in each case one study indicating that hypertension, liver cirrhosis, COPD and cardiac operations are predisposing factors for superficial infection after THA. Amongst others, these comorbidities are considered in the Charlson Comorbidity Index [21], which can be used to quantify the prognosis of patients to suffer a PJI after total joint arthroplasty.

Another study reported that dyspnoea predisposes patients for superficial infection after THA. Shortness of breath may lead to decreased oxygen delivery to the healing tissues, impairing effective tissue repair, wound healing and increasing susceptibility to infection.

Although it has been reported that patients with chronic kidney disease are likely to have higher rates of infection after THA, Miric et al. [12] could not confirm these results. The authors explain their results by pointing out, that previous studies have almost exclusively focused on patients with end-stage kidney disease, while their own study was focused on patients with earlier stages of chronic kidney disease.

Even though we found that the most prominent risk factor was BMI, followed by age, ASA class $>2$ and diabetes mellitus, this does not mean that they are the most severe risk factors for superficial infection, but currently the most investigated conditions. Obesity and diabetes for example are two of the most common disorders worldwide, with continuously rising numbers. In both cases, patients are more likely to undergo total joint arthroplasty.

Conditions represented with only one study might simply have a lower prevalence in patients with THA and therefore cases are sparsely investigated. Although underrepresented by the number of studies, these factors still might pose a more severe impact on the predisposition to superficial infections compared to BMI or diabetes mellitus.

\section{Conclusions}

We performed a literature search exclusively on patient-related risk factors for superficial SSI after total hip replacements. Until now, the studies related to this topic are rare. The current data situation to be found in literature is unsatisfactory, but the outcome of this study can be considered as suggestion to observe patients with comorbidities such as obesity, age, ASA class $>2$, diabetes mellitus, HIV, hypertension, chronic obstructive pulmonary disease (COPD), previous cardiac surgery, dyspnea, corticosteroid use and hepatitis $\mathrm{C}$. To guarantee the best possible outcome after the surgery, it is advisable to treat these patients before performing a total hip replacement - if possible.

\section{Limitations}

Unfortunately, in some cases the analyzed studies lack adequate power to draw final conclusions about the impact of a given condition on the rate of superficial SSI. 
Furthermore, our research was impeded by a lack of precise definition between superficial infection and deep PJI or other reasons for readmission in the literature.

During our analysis, we focused only on studies that investigated patients who received a primary THA. Risk factors for revision arthroplasty may be different or could have a more severe impact, since patients that undergo revision arthroplasty are more likely to be infected. Moreover, often comorbidities accumulate and influence each other, which may in turn affect their impact on infection rate.

Another issue to be considered is that due to the set-up of our study some potential risk factors discussed in the literature were not detected[7,9], e.g. alcohol abuse, smoking, depression or rheumatoid arthritis.

While being fully aware of these limitations, we still recommend that patient and surgeon consider the potential patient-related risk factors that we listed in Table 1 . Although evidence is sparse, precaution may lead to a reduced superficial infection rate. Several patient-dependent measures can be taken into account prior to surgery. For example, weight reduction in obese patients, establishment of stable glucose blood levels in diabetes mellitus patients, cessation period in alcoholic patients or the use of antibiotic loaded bone cements in patients that are burdened with potential risk factors. Based on our findings, we do not recommend withholding surgery in predisposed patients who otherwise meet reasonable surgical conditions.

\section{REFERENCES}

[1] K.-D. Kühn. Management of periprosthetic joint infection A global perspective on diagnosis, treatment options, prevention strategies and their economic impact, Springer-Verlag, Berlin/Heidelberg, 2018.

[2] H. Gollwitzer. [Endoprosthesis infection: Russian roulette or preventable complication? A Guide to the Prevention of Periprosthetic Infections], ATOS News, Vol. 20, 9-12, 2012.

[3] M. K. Allami, W. Jamil, B. Fourie, V. Ashton, P. J. Gregg. Superficial incisional infection in arthroplasty of the lower limb. Interobserver reliability of the current diagnostic criteria, J Bone Joint Surg Br, Vol. 87, 1267-1271, 2005.

[4] CDC definitions. Definitions of nosocomical infections, Robert Koch-Istitut, 2011.

[5] P. E. Ochsner, O. Borens, P.-M. Bodler, I. Broger, G. Eich, F. Hefti, T. Maurer, H. Nötzli, S. Seiler, D. Suvà, A. Trampuz, I. Uçkay, M. Vogt, W. Zimmerli. [Infections of the musculoskeletal system. Basic principles, prophylaxis, diagnostic and therapy], Swiss orthopaedics, Bourg-en-Lavaux, 2015.

[6] V. Pilz, T. Hahnstein, R. Skripitz. Projections of primary hip arthroplasty in Germany until 2040, Acta Orthop, Vol. 89(3), 308-313, 2018.

[7] B. Zmistowski, P. Alijanipour. Periprosthetic joint infection of the hip and knee - risk factors for periprosthetic joint infection, Springer-Verlag, New York, 2014.

[8] A. Eka, A. F. Chen. Patient-related medical risk factors for periprosthetic joint infection of the hip and knee, Ann Transl Med, Vol. 3(16), 233, 2015.

[9] M. Jesenko, R. Windhager, A. Kontekakis, T. Hahnstein, K.-D. Kühn. Risk factors for periprosthetic joint infections following primary total hip arthroplasty, Universal Journal of Medical Science, Vol. 4(1), 38-44, 2015.

[10] Q. Naziri, M. R. Boylan, K. Issa, L.C. Jones, H. S. Khanuja, M. A. Mont. Does HIV infection increase the risk of perioperative complications after THA? A nationwide database study. Clin Orthop Relat Res, Vol. 473(2), 581-586, 2015 .

[11] H. Dale, I. Skråmm,H. L. Løwer, H. M. Eriksen, B. Espehaug, O. Furnes, F. E. Skjeldestad, L. I. Havelin, L. B. Engesaeter. Infection after primary hip arthroplasty: a comparison of 3 Norwegian health registers, Acta Orthop, Vol. 82(6), 646-654, 2011

[12] A. Miric, M. C. Inacio, R. S. Namba. The effect of chronic kidney disease on total hip arthroplasty, J Arthroplasty, Vol. 29(6), 1225-1230, 2014.

[13] R. E. Mednick, H. M. Alvi, V. Krishnan, F. Lovecchio, D. W. Manning. Factors affecting readmission rates following primary total hip arthroplasty, J Bone Joint Surg Am, Vol. 96, 1201-1209, 2014.

[14] A. M. Davis, A. M. Wood, A. C. Keenan, I. J. Brenkel, J. A. Ballantyne. Does body mass index affect clinical outcome post-operatively and at five years after primary unilateral total hip replacement performed for osteoarthritis?: A multivariate analysis of prospective data, J Bone Joint Surg Br, Vol. 93, 1178-1182, 2011.

[15] R. Iorio, K. M. Williams, A. J. Marcantonio, L. M. Specht, J. F. Tilzey, W. L. Healy. Diabetes mellitus, hemoglobin A1C, and the incidence of total joint arthroplasty infection, J Arthroplasty, Vol. 27, 726-729, 2012.

[16] M. M. Dowsey, P. F. Choong. Obesity is a major risk factor for prosthetic infection after primary hip arthroplasty, Clin Orthop Relat Res, Vol. 466, 153-158, 2008.

[17] T. N. Peel, M. M. Dowsey, J. R. Daffy, P. A. Stanley, P. F Choong, K. L.Buising. Risk factors for prosthetic hip and knee infections according to arthroplasty site, J Hosp Infect, Vol. 79(2), 129-133, 2011

[18] Y. H. Chee, K. H. Teoh, B. M. Sabnis, J. A. Ballantyne, I. J. Brenkel. Total hip replacement in morbidly obese patients with osteoarthritis: results of a prospectively matched study, J Bone Joint Surg Br, Vol. 92(8), 1066-1071, 2010.

[19] A. E. Pour, W. Y. Matar, S. M. Jafari, J. J.Purtill, M. S. Austin, J. Parvizi. Total joint arthroplasty in patients with hepatitis C, J Bone Joint Surg Am, Vol. 93(15), 1448-1454, 2011.

[20] L. Font-Vizcarra, E. Tornero, G. Bori, J. Bosch, J. Mensa, A. Soriano. Relationship between intraoperative cultures during hip arthroplasty, obesity, and the risk of early prosthetic joint infection: A prospective study of 428 patients, Int J Artif Organs, Vol. 34(9), 870-875, 2011.

[21] M. E. Charlson, P. Pompei, K. L. Ales, C. R. MacKenzie. A new method of classifying prognostic comorbidity in longitudinal studies: development and validation, J Chronic Dis, 40, 373-383, 1987. 